$\begin{array}{cc}\text { ACADEMIA ROMÂNĂ } & \text { Rev. Roum. Chim., } \\ \text { Revue Roumaine de Chimie } & \text { 2020,6), 617-622 } \\ \text { http://web.icf.ro/rrch/ } & \text { DOI: 10.33224/rrch.2020.65.6.13 }\end{array}$

\title{
SYNTHESIS, CRYSTAL STRUCTURES AND SOLID-STATE OPTICAL PROPERTIES OF TWO MANNICH DERIVATIVES OF 2',7'-DICHLOROFLUORESCEIN
}

\author{
Mihai RĂDUCĂ and Augustin M. MĂDĂLAN* \\ Inorganic Chemistry Department, Faculty of Chemistry, University of Bucharest, Dumbrava Rosie 23, 020464-Bucharest, Roumania
}

Synthesis, crystal structures and solid-state optical properties of two Mannich derivatives of 2',7'dichlorofluorescein are reported herein. The first derivative (MBFluo1), resulted by Mannich reaction from 2',7'-dichlorofluorescein, paraformaldehyde and $\mathrm{N}$-methylpiperazine, crystallizes in a zwitterionic form, while the second derivative (MBFluo2) was obtained in the non-charged spironolactone form from 2', 7'-dichlorofluorescein, paraformaldehyde and di-tert-buthyliminodiacetate. The emission spectra of MBFluo2 present two maxima at 420 and $550 \mathrm{~nm}$.

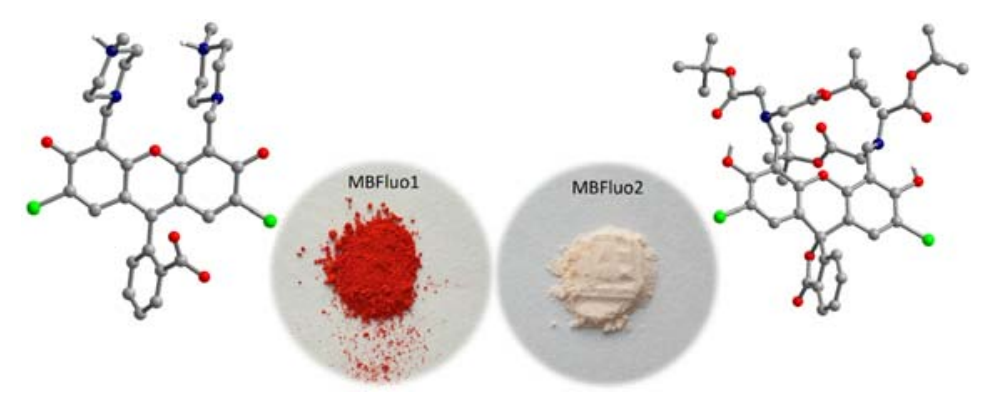

\section{INTRODUCTION}

Organic dyes are of great importance to society. They are widely used in various fields from textile and plastics industries to biomedical applications, production of photovoltaic materials and optical data storage devices. Among these pigments, the xanthenes (including fluoresceins and rhodamines) are fluorophores with excellent photophysical properties, such as high extinction coefficients, excellent quantum yields, great photostability, and relatively long emission wavelengths. ${ }^{1}$

Some of the valuable features of the fluorescein derivatives for biomedical applications are good water solubility, visible excitation and emission (an absorption maximum at $494 \mathrm{~nm}$ and emission maximum of $521 \mathrm{~nm}$ in water), and maximum brightness at physiological $\mathrm{pH}^{2}$ Spirocyclic derivatives of fluorescein are useful molecule based sensors because the ring-opening process leads to a turn-on fluorescence change. In solution, fluorescein derivatives are non-fluorescent when they exist in the lactone form, and the opening of the ring can induce colour changes and fluorescence enhancements (Scheme 1). In solid-state, the reduced separation between chromophores results in increase of nonradiative de-excitation pathways and quenching of the fluorescence.

The design of highly selective and efficient fluorescence sensors for the detections of chemically and biologically important species is currently of high interest for both, chemistry and biology. Fluorescein derivatives functionalized by Mannich reactions were used as fluorescence sensors for quantifying biological $\mathrm{Zn}$ (II) ions. ${ }^{3}$ The di(2-picolyl)amine moieties attached by Mannich

${ }^{*}$ Corresponding author: augustin.madalan@chimie.unibuc.ro 
reaction to 2',7'-dichlorofluorescein proved to be efficient chelators not only for $\mathrm{Zn}$ (II) ions but also for $\mathrm{Gd}(\mathrm{III})$ ions. $^{4}$ The presence of the basic nitrogen atoms on the Mannich base derivatives of the fluorescein offers the theoretical possibility of the existence of two forms of these compounds: the spironolactone form (a close ring form) and the zwitterionic form (an open ring form). A survey on the Cambridge Structural Database (CSD) revealed only the non-charged form of the Mannich derivatives of 2',7'-dichlorofluorescein in the crystal structures reported. ${ }^{3 a-d, 5}$ In this paper we report synthesis, crystal structures and solid-state optical properties of two Mannich derivatives of 2',7'-dichlorofluorescein.

\section{RESULTS AND DISCUSSION}

The first Mannich derivative (MBFluo1) was prepared analogously to the literature from $2^{\prime}, 7^{\prime}-$ dichlorofluorescein, paraformaldehyde and $\mathrm{N}$ methylpiperazine, while the second derivative (MBFluo2) was also obtained by Mannich reaction from 2',7'-dichlorofluorescein, paraformaldehyde and di-tert-buthyliminodiacetate. In Scheme 2 are presented the spironolactone forms of the two Mannich derivatives.
For both compounds crystals suitable for X-ray diffraction were obtained by slow evaporations. The structural characterization by X-ray diffraction on single crystal showed that MBFluo1 is present in crystal in the zwitterionic form (Figure 1). This is the first report of a crystal structure containing the zwitterionic form (open ring form) of a Mannich derivative of fluorescein. MBFluo1 crystallizes in the monoclinic P21/c space group with one organic molecule and eight water molecules in the asymmetric unit. This Mannich base derivative is crystallized in the zwitterionic form with two amino groups protonated (the nitrogen atoms N2 and N4). The bond lengths $\mathrm{C} 3-$ $\mathrm{O} 1$ and $\mathrm{C} 11-\mathrm{O} 3,1.259(6)$ and 1.248(7) $\AA$ respectively, are corresponding rather to a double bond $\mathrm{C}=\mathrm{O}$ showing that both phenol groups are deprotonated. The carboxylate group has the two $\mathrm{C}-\mathrm{O}$ bond lengths very similar, $\mathrm{C} 20-\mathrm{O} 4=1.242(8)$ and $\mathrm{C} 20-\mathrm{O} 5=1.251(8) \AA$. The carboxylate group is twisted out of the plane of the aromatic ring making with this a dihedral angle of $41.3^{\circ}$. The dihedral angle between the mean plans of the phenyl ring and xanthene fragment is $63.2^{\circ}$. The two N-methylpiperazine fragments are located one on each side of the plane of the xanthene fragment (Figure 1b).

\section{Fluorescein}<smiles>O=C1OC2(c3ccc(O)cc3Oc3cc(O)ccc32)c2ccccc21</smiles><smiles>O=C(O)c1ccccc1-c1c2ccc(=O)cc-2oc2cc(O)ccc12</smiles>

Scheme 1

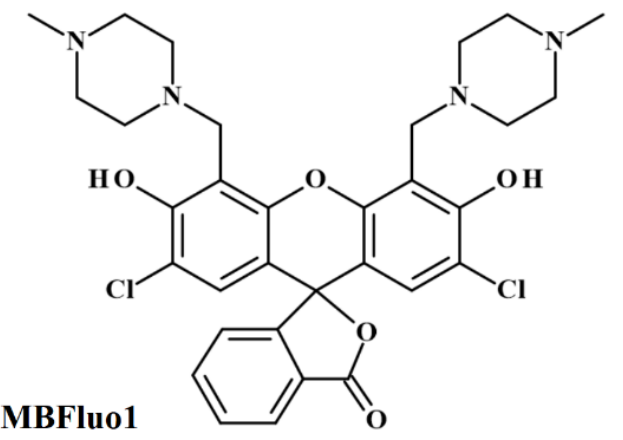

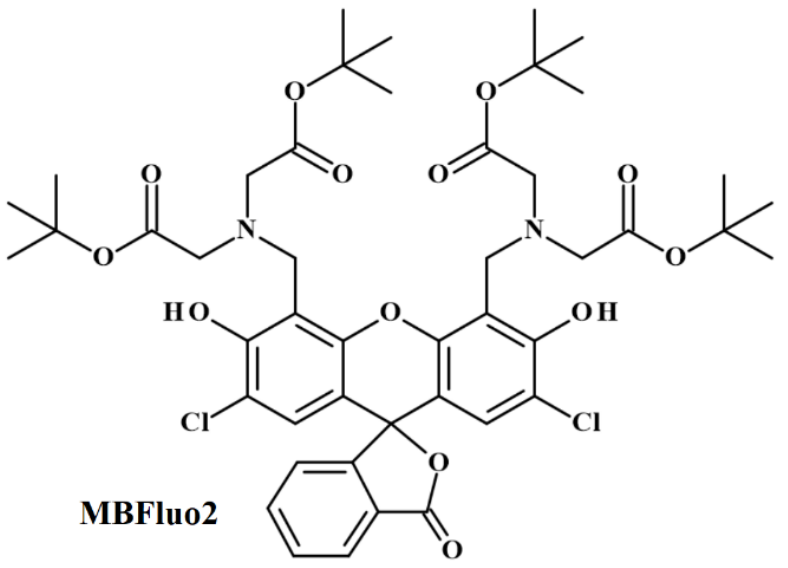



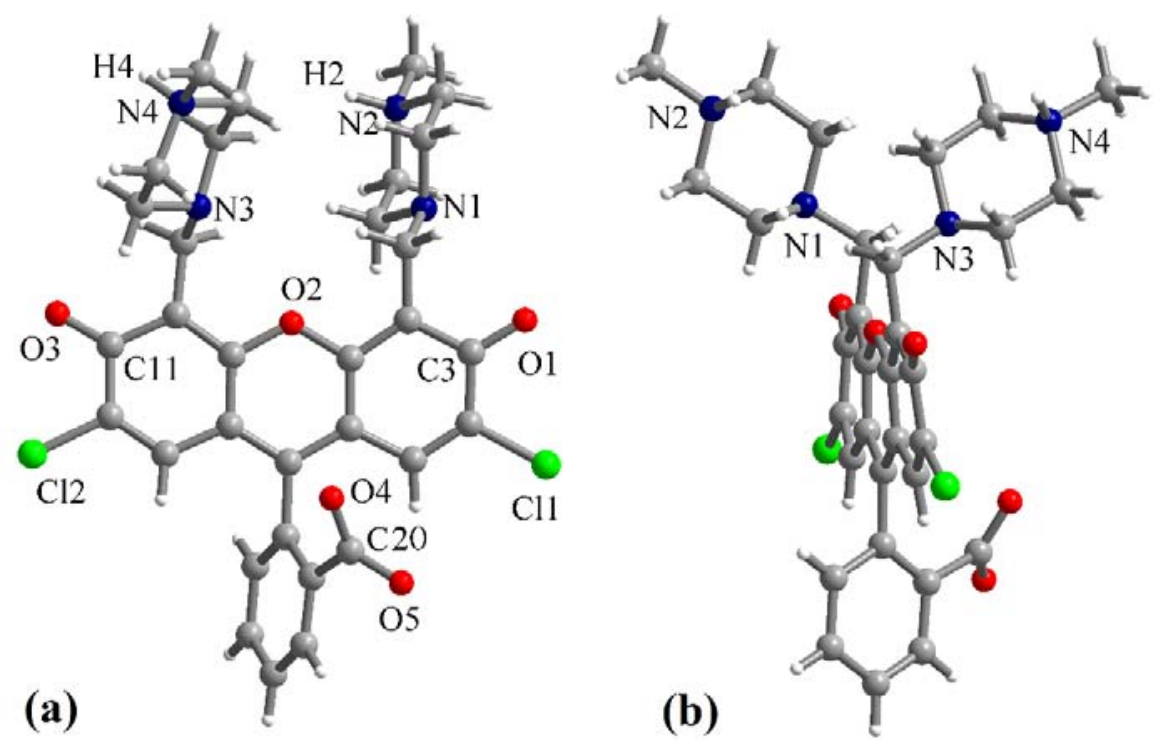

Fig. 1 - Perspective views of the molecular structure of MBFluo1 determined by X-ray diffraction.

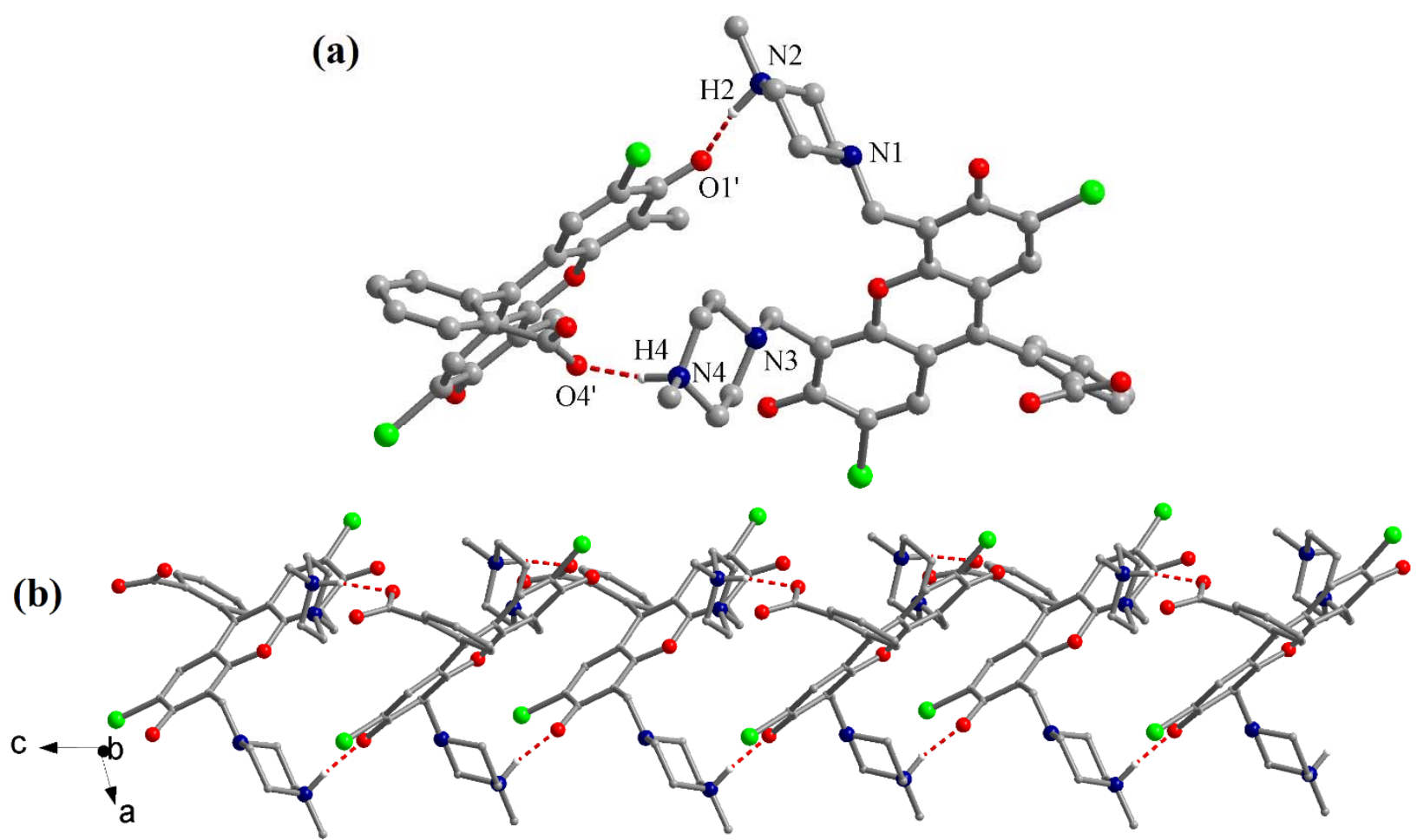

Fig. 2 - Perspective views in crystal MBFluo1 showing: (a) the hydrogen interactions established by the protonated amino groups (symmetry code: ' $=\mathrm{x}, 1.5-\mathrm{y},-0.5+\mathrm{z}$, the N-methylpiperazine fragments of the acceptor molecule and the hydrogen atoms not involved in hydrogen interactions where omitted for clarity) and (b) the supramolecular chain generated by hydrogen bonding.

The protonated amino groups of the two Nmethylpiperazine fragments (corresponding to the nitrogen atoms $\mathrm{N} 2$ and N4) are involved as donors in hydrogen interactions with the carboxylate group and one quinone group of a neighbouring molecule (Figure 2a). The distances (N2-)H2 $\cdots \mathrm{O} 1$ ' and (N4-)H4어' (symmetry code: ' $=\mathrm{x}, 1.5-\mathrm{y}$, $0.5+\mathrm{z})$ are 1.77 and $1.82 \AA$, while the corresponding $\mathrm{N} 2-\mathrm{H} 2 \cdots \mathrm{O} 1{ }^{\prime}$ and $\mathrm{N} 4-\mathrm{H} 4 \cdots \mathrm{O} 4$ ' angles are 153.0 and $170.1^{\circ}$, respectively. This type of non-covalent interactions between the organic molecules extends along the crystallographic $c$ axis generating supramolecular chains (Figure 2b). The hydrogen interactions pattern of the crystallization water molecules will not be described here because three of the eight water molecules are disordered on six independent crystallographic positions with site occupancy factors of 0.5 and the hydrogen 
interactions between these molecules cannot be assigned precisely.

The second Mannich derivative described herein, MBFluo2, was obtained by reacting 2',7'dichlorofluorescein, paraformaldehyde and di-tertbuthyliminodiacetate. The MBFluo2 is the tetratert-buthyl ester of 2',7'-dichlorocalcein. The 2',7'-dichlorocalcein was used for sensing $\mathrm{Cu}(\mathrm{II})$ ions $^{6}$, cyanide ${ }^{7}$ or to create near-infrared luminescent probes by coordination to lanthanide ions. ${ }^{8}$ MBFluo2 crystallizes in the monoclinic $\mathrm{C} 2 / \mathrm{c}$ space group as non-charged spironolactone form (Figure 3). The $\mathrm{O} 2$ atom lies on a two-fold rotation axis and, as a consequence of this fact, the phenyl ring and the spironolactone fragment are disordered on two crystallographic positions (in
Figure 3 is depicted only one of these positions for clarity). The $\mathrm{C} 3-\mathrm{O} 1$ bond length of the phenol group is 1.344(4) $\AA$ and the $\mathrm{OH}$ group is involved in intramolecular hydrogen bonding with the amino nitrogen atom. The distance $(\mathrm{O} 1-) \mathrm{H} 1 \cdots \mathrm{N} 1$ is $2.12 \AA$, while the corresponding $\mathrm{O} 1-\mathrm{H} 1 \cdots \mathrm{N} 1$ angle is $143.5^{\circ}$. The $\mathrm{C} 14-\mathrm{O} 3, \mathrm{C} 14-\mathrm{O} 4$ and $\mathrm{C} 7-\mathrm{O} 3$ bond lengths are: $1.350(11), 1.237(13)$ and 1.505(8) $\AA$, respectively. The bulky di-tertbuthyliminodiacetate groups have one arm on each side of the xanthene core.

Spectroscopic studies. The electronic spectra of the two compounds have been acquired over a wavelength range from 200 to $1800 \mathrm{~nm}$ on solid sample (using the diffuse reflectance technique).

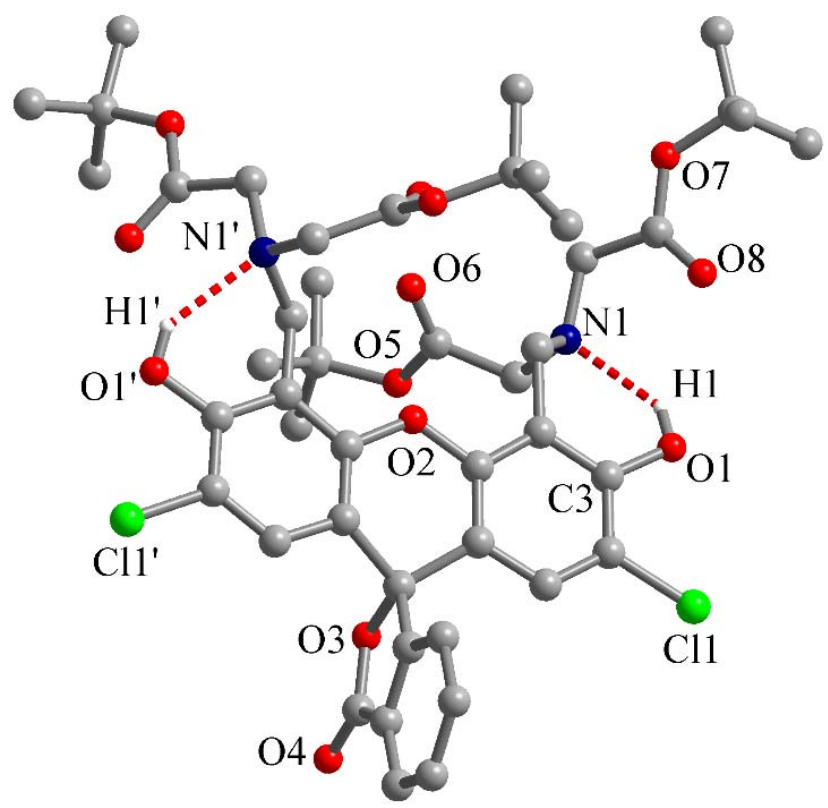

Fig. 3 - Perspective view of the molecular structure of MBFluo2 determined by X-ray diffraction.

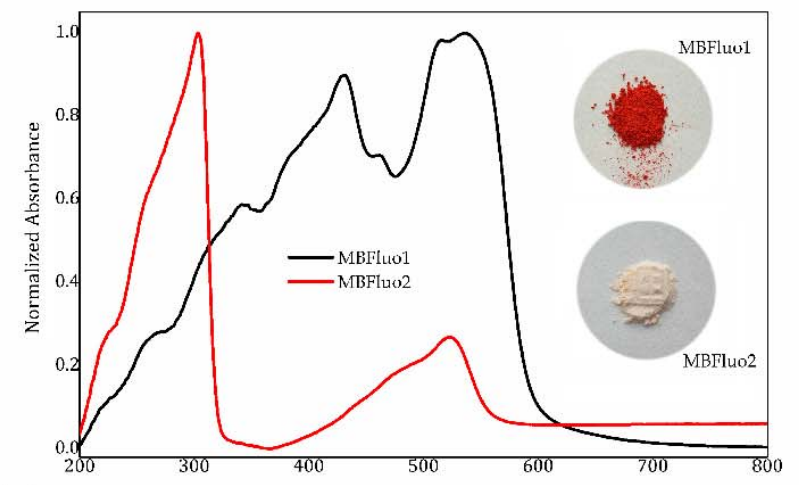

(a)

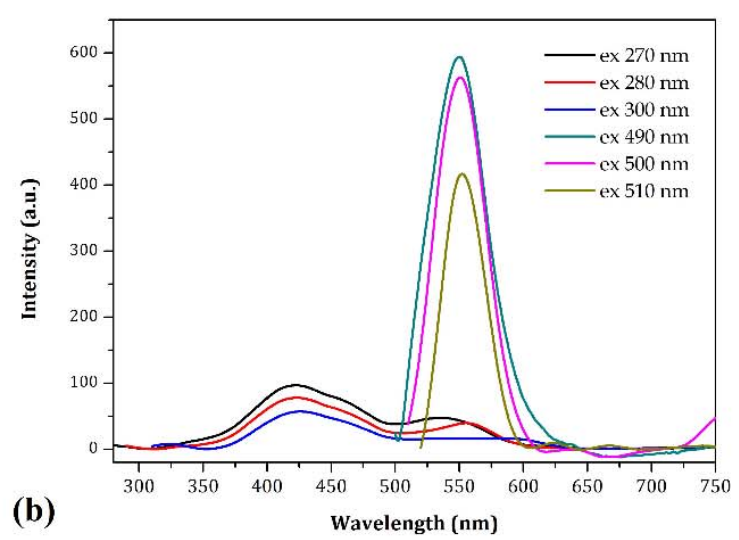

Fig. 4 - (a) The solid-state electronic spectra of MBFluo1 and MBFluo2 (the inset shows the colours of the two compounds) and (b) the emission spectra of MBFluo2 in solid-state. 

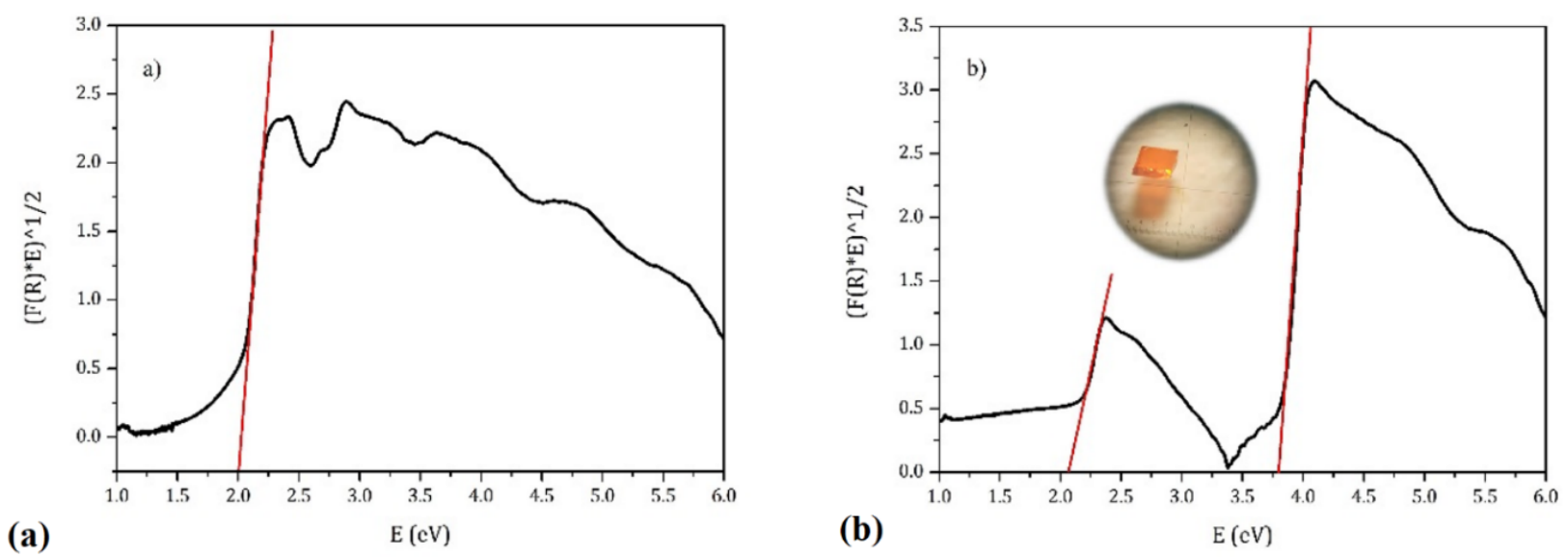

Fig. 5 - Tauc plots $^{9}$ for MBFluo1 (a) and MBFluo2 (b). The band gaps are calculated by extrapolating the linear regions of the plots to the $x$-axis (shown in red). The inset: the colour of a MBFluo2 crystal.

MBFluo1 exhibits absorption maxima at 431 and $536 \mathrm{~nm}$ and several shoulders, while MBFluo2 presents two maxima at 303 and $524 \mathrm{~nm}$, respectively (Figure 4a). In the case of MBFluo2, the UV absorption band has a much higher intensity than that one from visible region. There is a strong dependence between the colour of a material and the value of band gap: photons with energy lower than the band gap will be reflected, while photons with higher energy will be absorbed. The values of band gaps of the two fluorescein derivatives were measured using the solid-state $\mathrm{UV} / \mathrm{Vis}$ spectroscopy data.

The Tauc plots ${ }^{9}$ for MBFluo1 and MBFluo2 are presented in Figure 5. In the case of MBFluo1 the measured band gap, $2.0 \mathrm{eV}$, corresponds to the zwitterionic form, ${ }^{10}$ while for MBFluo2 the lines crossing the $x$-axis at $2.1 \mathrm{eV}$ and $3.8 \mathrm{eV}$ correspond to the bad gap of the zwitterionic form and to the band gap of the spirolactone form, respectively. The last one is colourless, ${ }^{10}$ and the light pink colour of the material can be associated with the presence of a fraction containing the zwitterionic form of the Mannich derivative (see the insets of Figures $4 a$ and $5 b$ ).

The luminescent properties of compounds have been investigated at room temperature in the solid state. MBFluo1 presents poor emission in visible region, low intensity bands being observed in the emission spectra. The room temperature photoluminescence of compound MBFluo2 was investigated using different wavelengths for excitation in the $270-510 \mathrm{~nm}$ range (Figure $4 \mathrm{~b}$ ). For MBFluo2 the emission spectra present two maxima at $420 \mathrm{~nm}$ (the highest intensity of the emission was obtained upon excitation at $270 \mathrm{~nm}$ ) and $550 \mathrm{~nm}$ (the highest intensity of the emission was obtained upon excitation at $490 \mathrm{~nm}$ ), respectively. The absence of the fluorescence in the case of MBFluo1 is caused probably by aggregation effects due to the fact that in solid is present mainly the zwitterionic open-ring form. ${ }^{11}$ In MBFluo2 sample there is only a small fraction of the zwitterionic form diluted in the spirolactone form.

\section{EXPERIMENTAL}

\section{Materials and methods}

\section{Synthesis of the Mannich derivatives}

All the chemicals used as well as all the solvents were purchased from commercial sources. All manipulations were performed under aerobic conditions.

MBFluo1 was prepared analogously to the literature. ${ }^{5}$ Crystals suitable for X-ray diffraction were obtained by slow evaporation from a mixture of isopropanol, acetonitrile and dimethylformamide.

MBFluo2. $0.7788 \mathrm{~g}$ of 2',7'-dichlorofluorescein (1.94 mmol), $1 \mathrm{~g}$ of di-tert-buthyliminodiacetate $(4.08 \mathrm{mmol})$ and $0.1281 \mathrm{~g}$ of paraformaldehyde $(4.27 \mathrm{mmol})$ were dissolved in $20 \mathrm{~mL}$ absolute ethanol. The mixture was refluxed for $48 \mathrm{~h}$ yielding a white precipitate of desired compound which turned slightly pink. The precipitate was washed with $40 \mathrm{~mL}$ cold absolute ethanol and 20 $\mathrm{ml}$ diethylether and it was dried. For further purification it was crystallized from a mixture of solvents isopropanol and tetrahydrofuran $(1: 1)$ obtaining light orange crystals suitable for $\mathrm{X}$-ray diffraction. The yield was $86 \%$.

Elemental analysis: Calcd.: C, 59.16; H, 6.26; N, 3.0\%. Found: C, 59.23, H, 6.03, N, 3.12\%. Selected IR data (KBr pellet, $\left.\mathrm{cm}^{-1}\right)$ : 3240(bw), 2978(m), 1776(s), 1732(vs), 1628(w), 1477(m), 1433(m), 1369(s), 1248(s), 1215(s), 1148(vs), 1094(m), 1028(w), 980(w), 895(w), 874(m), 851(w), 698(m), 565(w).

\section{X-Ray crystallography}

X-ray diffraction measurements were performed on a STOE IPDS II diffractometer, operating with Mo-K $\alpha(\lambda=0.71073 \AA)$ $\mathrm{X}$-ray tube with graphite monochromator. The structures were solved by direct methods (using SHELXS-2013 crystallographic software) and refined by full-matrix least squares techniques based on $F^{2}$. The non-H atoms were refined with anisotropic displacement parameters. Calculations were performed using SHELXL-2018 crystallographic software package. A summary of the crystallographic data and the structure refinement for the two compounds are given in Table 1. CCDC reference numbers: 1993701-1993702. 
Table 1

Crystallographic data, details of data collection and structure refinement parameters for MBFluo1 and MBFluo2

\begin{tabular}{lll}
\hline Compound & MBFluo1 & MBFluo2 \\
\hline Chemical formula & $\mathrm{C}_{32} \mathrm{H}_{60} \mathrm{Cl}_{2} \mathrm{~N}_{4} \mathrm{O}_{13}$ & $\mathrm{C}_{46} \mathrm{H}_{56} \mathrm{Cl}_{2} \mathrm{~N}_{2} \mathrm{O}_{14.5}$ \\
$M\left(\mathrm{~g} \mathrm{~mol}^{-1}\right)$ & 779.74 & 939.82 \\
Temperature, $(\mathrm{K})$ & $293(2)$ & $293(2)$ \\
Wavelength, $(\AA)$ & 0.71073 & 0.71073 \\
Crystal system & Monoclinic & Monoclinic \\
Space group & $P 21 / c$ & $C 2 / c$ \\
$a(\AA)$ & $12.1595(8)$ & $23.8541(16)$ \\
$b(\AA)$ & $24.2155(11)$ & $13.0875(10)$ \\
$c(\AA)$ & $13.6395(8)$ & $21.4203(16)$ \\
$\alpha\left({ }^{\circ}\right)$ & 90 & 90 \\
$\beta\left({ }^{\circ}\right)$ & $105.374(5)$ & $130.893(4)$ \\
$\gamma\left({ }^{\circ}\right)$ & 90 & 90 \\
$V\left(\AA^{3}\right)$ & $3872.4(4)$ & $5055.1(7)$ \\
$\mathrm{Z}$ & 4 & 4 \\
$D_{\mathrm{c}}\left(\mathrm{g} \mathrm{cm}^{-3}\right)$ & 1.337 & 1.235 \\
$\mu\left(\mathrm{mm}^{-1}\right)$ & 0.234 & 0.192 \\
$\mathrm{~F}(000)$ & 1672 & 1984 \\
Goodness-of-fit on $F^{2}$ & 1.041 & 1.146 \\
Final $R 1, w R_{2}[I>2 \sigma(I)]$ & $0.0801,0.1536$ & $0.0715,0.1850$ \\
$R 1, w R_{2}$ (all data) & $0.1980,0.2080$ & $0.1001,0.2015$ \\
Largest diff. peak and hole $\left(\mathrm{e} \AA^{-3}\right)$ & $0.356,-0.336$ & $0.259,-0.252$ \\
\hline
\end{tabular}

Physical measurements. The IR spectra ( $\mathrm{KBr}$ pellets) were collected on a Bruker Tensor 37 spectrophotometer in the 4000$400 \mathrm{~cm}^{-1}$ range. The UV-Vis-NIR spectra (diffuse reflectance technique) were recorded on a JASCO V-670 spectrophotometer. The fluorescence spectra were collected on a JASCO FP-6500 spectrofluorometer. Elemental analysis of $\mathrm{C}, \mathrm{N}$ and $\mathrm{H}$ were performed on a EuroEA3000 Elemental Analyzer.

\section{CONCLUSIONS}

In this article, we describe the synthesis and characterization of two Mannich derivatives of 2',7'-dichlorofluorescein. Optical properties of the compounds depend on the structure of the fluorescein core, which can exist in the spironolactone form (a close ring non-charged form) or in the zwitterionic form (an open ring form). Since Mannich derivatives contain basic nitrogen atoms (potential acceptors of the protons derived from the acidic phenol groups), both forms can be obtained for these compounds. Herein we report the first crystal structure of a Mannich derivative of fluorescein in the zwitterionic form.

Acknowledgements. Financial support from the UEFISCDI (Project PN-III-P4-ID-PCE-2016-0442 nr. 89/2017) is gratefully acknowledged.

\section{REFERENCES}

1. X. Chen, T. Pradhan, F. Wang, J. S. Kim and J. Yoon, Chem. Rev., 2012, 112, 1910.

2. M. S. T. Gonçalves, Chem. Rev., 2009, 109, 190.

3. a) X. Zhang, D. Hayes, S. J. Smith, S. Friedle and S. J. Lippard, J. Am. Chem. Soc., 2008, 130, 15788; b) B. A. Wong, S. Friedle and S. J. Lippard, J. Am. Chem. Soc., 2009, 131, 7142; c) E. M. Nolan, J. W. Ryu, J. Jaworsky, R. P. Feazell, M. Sheng and S. J. Lippard, J. Am. Chem. Soc., 2006, 128, 15517; d) B. A. Wong, S. Friedle and S. J. Lippard, Inorg. Chem., 2009, 48, 7009; (e) L. E. McQuade and S. J. Lippard, Inorg. Chem., 2010, 49, 9535.

4. D. Dong, X. Jing, X. Zhang, X. Hu, Y. Wu and C. Duan, Tetrahedron, 2012, 68, 306.

5. K. M. K. Swamy, H. N. Kim, J. H. Soh, Y. Kim, S. J. Kim and J. Yoon, Chem. Commun., 2009, 1234.

6. E. J. Jun, J.-A. Kim, K. M. K. Swamy, S. Park and J. Yoon, Tetrahedron Lett., 2006, 47, 1051.

7. S.-Y. Chung, S.-W. Nam, J. Lim, S. Park and J. Yoon, Chem. Commun., 2009, 2866.

8. T. Terai, Y. Urano, S. Izumi, H. Kojima and T. Nagano, Chem. Commun., 2012, 48, 2840.

9. J. Tauc, R. Grigorovici and A. Vancu, Phys. Status Solidi., 1966, 15, 627.

10. M. Arhangelskis, M. D. Eddleston, D. G. Reid, G. M. Day, D. K. Bucar, A. J. Morris and W. Jones, Chem. Eur. $J ., 2016,22,10065$.

11. a) A. P. Green and A. R. Buckley, Phys. Chem. Chem. Phys., 2015, 17, 1435; b) S. Feng, S. Gong and G. Feng, Chem. Commun., 2020, 56, 2511. 